\title{
ANÁLISIS AMBIENTAL DE LA MOVILIDAD Y EL CONSUMO DE PAPEL EN LOS ESTUDIANTES UNIVERSITARIOS
}

\section{ENVIRONMENTAL ANALYSIS OF UNIVERSITY STUDENTS' MODE OF TRANSPORTATION AND PAPER CONSUMPTION}

\author{
María José Fernández \\ Universidad de Buenos Aires \\ Facultad de Ciencias Económicas \\ Buenos Aires, Argentina \\ mariajfernandez@economicas.uba.ar
}

\author{
Verónica García Fronti \\ Universidad de Buenos Aires \\ Facultad de Ciencias Económicas \\ Buenos Aires, Argentina \\ vgarciafronti@economicas.uba.ar
}

\author{
Andrea Parma \\ Universidad de Buenos Aires \\ Facultad de Ciencias Económicas \\ Buenos Aires, Argentina \\ andreaparma38@gmail.com
}

Fecha de recepción: 09/09/2019 - Fecha de revisión: 03/12/2019 - Fecha de aprobación: 19/12/2019

DOI: https://doi.org/10.36995/j.visiondefuturo.2020.24.02.004.es

\section{RESUMEN}

Las actividades que se desarrollan en las organizaciones educativas tienen un determinado impacto ambiental. Analizar el efecto de las mismas sobre el medio ambiente permite determinar y priorizar acciones para minimizarlo. Una forma de cuantificarlo es mediante el indicador ambiental Huella Ecológica que tiene un alto potencial como herramienta de concientización.

Este tipo de organizaciones presentan un alto consumo de papel. Además, la movilidad de la comunidad hacia las sedes en las cuales se realizan las actividades académicas involucra un uso intensivo de transporte (trenes, subte, colectivos, bicicletas y autos particulares).

Con el objetivo de calcular el indicador Huella Ecológica se realizó una encuesta a estudiantes de la asignatura Análisis Matemático II de la Facultad de Ciencias Económicas (FCE) de la Universidad de Buenos Aires (UBA) para determinar el consumo de papel y el tipo de movilidad utilizada para asistir a clase. Con estos datos, se calculó el impacto ambiental de dicha actividad.

En este trabajo se describe la metodología utilizada y se presentan los resultados obtenidos sobre estos dos aspectos ambientales: consumo de papel y transporte estudiantil.

PALABRAS CLAVE: Gestión Ambiental Universitaria; Huella Ecológica; Indicadores.

"Visión de Futuro" Año 17, Volumen No 24 No 2, Julio - Diciembre 2020 - Pág 125 - 145

URL de la Revista: http://visiondefuturo.fce.unam.edu.ar/index.php/visiondefuturo/index

URL del Documento: https://visiondefuturo.fce.unam.edu.ar/index.php/visiondefuturo/issue/view/18

ISSN 1668 - 8708 - Versión en Línea

E-mail: revistacientifica@fce.unam.edu.ar 


\section{ABSTRACT}

The activities undertaken in educational organizations have a certain environmental impact. Analyzing their effect on the environment makes it possible to determine and prioritize actions aimed at minimizing it. One way to quantify such effect is by means of the Ecological Footprint environmental indicator, which has a high potential when used as an awareness-raising tool.

These types of organizations have a high level of paper consumption. In addition, students commute to their campuses, thus involving an intensive use of transport systems (trains, subway, buses, bicycles, and private cars).

In order to calculate the Ecological Footprint indicator, a survey responded to by students of the 'Mathematical Analysis Il' subject at the School of Economics of the Universidad de Buenos Aires was carried out, so as to determine their paper consumption and the mode of transportation used to attend their classes. Based on these data, the environmental impact of this activity was calculated.

This paper describes the methodology used and presents the results obtained for these two environmental aspects: paper consumption and students' transport.

KEY WORDS: University environmental management; Ecological Footprint; Indicators.

\section{INTRODUCCIÓN}

El indicador ambiental Huella Ecológica permite analizar e indagar sobre el impacto ambiental de las actividades humanas desarrolladas a nivel mundial, nacional u organizacional. Si bien es un indicador no muy utilizado a este último nivel, en el marco de una institución universitaria tiene un alto potencial al utilizarlo como herramienta de concientización. El mismo permite conocer cómo impactan cada una de las actividades universitarias sobre el medio ambiente.

Las universidades que más utilizaron este indicador fueron las angloparlantes. También lo han aplicado algunas universidades españolas, como el caso de la Universidad de Málaga (Universidad de Málaga, 2016).

En este tipo de organizaciones las actividades allí desarrolladas generan un alto consumo de papel y la movilidad de la comunidad universitaria hacia las sedes involucra un uso intensivo de transporte (trenes, subte, colectivos, bicicletas y autos particulares).

Con el objetivo de calcular el indicador Huella Ecológica se realizó durante el año 2016 una encuesta a los estudiantes de la Facultad de Ciencias Económicas de la Universidad de Buenos Aires que estaban cursando la asignatura Análisis Matemático II, de esta forma se determinaron los consumos de papel y el tipo de movilidad de un grupo de estudiantes universitarios.

"Visión de Futuro" Año 17, Volumen No 24 No 2, Julio - Diciembre 2020 - Pág 125 - 145

URL de la Revista: http://visiondefuturo.fce.unam.edu.ar/index.php/visiondefuturo/index

URL del Documento: https://visiondefuturo.fce.unam.edu.ar/index.php/visiondefuturo/issue/view/18

ISSN 1668 - 8708 - Versión en Línea

E-mail: revistacientifica@fce.unam.edu.ar 
En una primera etapa, se indagó respecto al indicador de sustentabilidad Huella Ecológica y la factibilidad de aplicación en la FCE-UBA (Fernandez et al., 2017). Se analizaron dos de los indicadores parciales que componen la Huella Ecológica: consumo de papel y transporte de los estudiantes. Se definió la metodología para el relevamiento y se diseñó una encuesta para los alumnos sobre sus hábitos de consumo de papel y su movilidad.

En este trabajo se avanzó sobre la segunda etapa y se presentan los resultados del primer trabajo de campo realizado a estudiantes.

\section{DESARROLLO}

\section{Metodología}

El enfoque utilizado para abordar este trabajo es el propuesto por Wackernagel y Rees (2001) para analizar la sustentabilidad de las actividades humanas. Busca visibilizar la presión que realizan sobre el ecosistema natural en el que se desarrollan. Para lograr esto los autores diseñaron un indicador biofísico denominado Huella Ecológica $(\mathrm{HE})$ y propusieron una metodología para su cálculo. La misma será utilizada en este artículo.

El indicador HE evalúa cuantitativamente el impacto de las actividades humanas en término de superficies productivas de los ecosistemas. Así la HE expresa el área necesaria para producir los recursos consumidos y asimilar los residuos generados por una población en un período de tiempo y lugar determinado. Es un indicador de índice único (integrado) y descriptivo que revela cuantas hectáreas de naturaleza se necesitan para abastecer los procesos productivos y para absorber los desechos que genera una economía (Wackernagel y Rees, 2001).

La Huella Ecológica se expresa, generalmente en hectáreas globales por persona y año, por lo tanto, dependerá de dos factores, por un lado, el estilo de vida del individuo analizado, es decir sus hábitos de consumo y por otro lado el sistema ecológico necesario para generar esos recursos y asimilar los residuos generados.

Wackernagel y Rees (2001) definieron cinco categorías de consumo, es decir cinco formas en que utilizamos los recursos naturales: alimentación, vivienda, transporte, artículos de consumo y servicios. De esta forma el indicador HE está formado por indicadores parciales de acuerdo con la categoría que se desea analizar. En este trabajo estudiaremos dos indicadores parciales que componen la HE, una asociada a la movilidad de los estudiantes hacia la sede universitaria y otra asociada al consumo de papel para estudiar. Si bien ambas categorías determinan una Huella Ecológica parcial, las metodologías de cálculo difieren conceptualmente. A continuación, se describen brevemente ambas metodologías.

\footnotetext{
"Visión de Futuro" Año 17, Volumen No 24 Nº 2, Julio - Diciembre 2020 - Pág 125 - 145

URL de la Revista: http://visiondefuturo.fce.unam.edu.ar/index.php/visiondefuturo/index

URL del Documento: https://visiondefuturo.fce.unam.edu.ar/index.php/visiondefuturo/issue/view/18

ISSN 1668 - 8708 - Versión en Línea

E-mail: $\underline{\text { revistacientifica@fce.unam.edu.ar }}$
} 


\section{Huella Ecológica parcial movilidad de los estudiantes}

Para el cálculo de HE parcial movilidad se eligió cómo método el que estima el área de suelo necesaria para secuestrar $\mathrm{CO} 2$ emitido por la combustión de los diferentes medios de transporte utilizados (Wackernagel y Rees, 2001).

En este método se tiene en cuenta que los bosques acumulan $\mathrm{CO} 2$ a una tasa muy elevada a lo largo de períodos que van desde 50 hasta 80 años. Los datos sobre las productividades boscosas promedio de los bosques templados, boreales y tropicales, muestran que los bosques promedio pueden acumular aproximadamente 1,8 toneladas de CO2 por hectárea por año (Wackernagel y Rees, 2001).

De esta forma, la HE per cápita de movilidad (HE movilidad) se determinará calculando primero la cantidad de emisiones de $\mathrm{CO} 2$ debido al consumo de combustible fósil y luego se dividirán estas emisiones por el factor de fijación (1).

Huella ecológica movilidad per cápita (ha/año) $=$ Emisión (ton $\mathrm{CO}_{2}$ )

$$
\text { Fijación Media } \mathrm{CO}_{2} \text { (ton } \mathrm{CO}_{2} / \text { ha/año) }
$$

\section{HE parcial consumo de papel}

El papel para imprimir o escribir que utilizamos proviene del consumo de un recurso natural renovable como es la celulosa. La celulosa puede ser de origen forestal o vegetal. En ambos casos, los principales insumos para su fabricación son, además de la celulosa, la energía y el agua consumidas en el proceso de fabricación del papel.

El indicador Huella Ecológica de papel (HE papel) se basa en la idea de que para cada tonelada de papel consumido se requiere una cierta cantidad de suelo para proporcionar el recurso necesario (celulosa). En este caso, no se analiza el proceso de producción del papel, por lo que no se incluye en el indicador consideraciones sobre el consumo de energía y agua.

Para calcular el indicador HE per cápita papel lo primero que se debe obtener son las toneladas de papel consumido por cada estudiante durante un año, para esto, se realizó una encuesta a los alumnos consultándoles sobre sus principales consumos de papel para estudiar: uso de cuadernos tipo universitarios, impresiones propias y fotocopias. De esta forma calcula el consumo promedio anual per cápita de los estudiantes encuestados.

El siguiente paso es estimar la superficie de suelo per cápita para la producción del papel consumido. Para esto se divide el consumo anual promedio del papel entre su productividad anual promedio. El cálculo de la HE papel per cápita se realiza de la siguiente forma (2):

HE papel per cápita (ha/año) = Consumo anual per capital papel (tonelada) Productividad anual (tonelada / ha)

Para el valor de la productividad de papel en Argentina se usaron los datos de la localidad de Tandil en el trabajo: Indicador espacial del metabolismo urbano. Huella Ecológica de la ciudad de

"Visión de Futuro" Año 17, Volumen N²4 N², Julio - Diciembre 2020 - Pág 125 - 145

URL de la Revista: http://visiondefuturo.fce.unam.edu.ar/index.php/visiondefuturo/index

URL del Documento: https://visiondefuturo.fce.unam.edu.ar/index.php/visiondefuturo/issue/view/18

ISSN 1668 - 8708 - Versión en Línea

E-mail: revistacientifica@fce.unam.edu.ar 
Tandil, Argentina de Guerrero y Guiñirgo del año 2008. En ese trabajo, se considera que el pino es el árbol que se utiliza para producir la pasta de celulosa.

\section{Cálculo HE parciales}

El procedimiento para calcular ambos indicadores parciales permite tomar conciencia sobre el impacto producido por los estudiantes al movilizarse y consumir papel para realizar sus actividades universitarias.

Como se ha mencionado al describir el método de cálculo para las HE parciales, para conocer los hábitos de transporte y consumo de los estudiantes se realizó en el año 2016 una encuesta en cuatro cursos de Análisis Matemático II de la Facultad de Ciencias Económicas de la Universidad de Buenos Aires.

La encuesta se administró a través de un formulario accesible desde el campus virtual institucional de la Facultad. Los participantes del aula virtual son sólo los alumnos inscriptos en la materia y los docentes a cargo de la misma. La encuesta fue anónima y su realización optativa. Antes de su incorporación en el campus el docente a cargo de cada curso explicó cuál era el objetivo de la encuesta y el concepto de HE.

La encuesta constó de 12 preguntas. Las primeras 6 preguntas relacionadas con la caracterización del alumno encuestado, las preguntas 7 a 9 vinculadas con la movilidad y el resto (preguntas 10 a 12) al consumo de papel (Figura 1).

\footnotetext{
"Visión de Futuro" Año 17, Volumen No 24 N² 2, Julio - Diciembre 2020 - Pág 125 - 145

URL de la Revista: http://visiondefuturo.fce.unam.edu.ar/index.php/visiondefuturo/index

URL del Documento: https://visiondefuturo.fce.unam.edu.ar/index.php/visiondefuturo/issue/view/18

ISSN 1668 - 8708 - Versión en Línea

E-mail: revistacientifica@fce.unam.edu.ar
} 
Figura $\mathbf{N}^{\circ} 1$. Formulario de encuesta

\section{ENCUESTA HUELLA ECOLÓGICA FCE - UBA}

Esta encuesta es anónima y corfidencisl Los resultados de is misms se utiliwarin con fines exdusivarnente acrodénicos en el morco de uns Imestigroión CMA / CMMBACE - IADCOM - FCE - UBA.

Agradecernos su colaboraciön.

Su respuesta es anorima.

+ celigatorio

1. Edad*

El valor dobe ser un nümero.

2. Carrera*

Actuario Econamia

Actuario Adoministracion

Economia

3. Sede *

Coedoba

O Panenai

4. ¿Trabajás? *

O si

No

5. Horario de cursada *

Mañana $(7.13 \mathrm{hs}$.

Tarde (13 19s:

Noche $[1923 \mathrm{hs})$

Fuente: Elaboración Propia

"Visión de Futuro" Año 17, Volumen No 24 N², Julio - Diciembre 2020 - Pág 125 - 145

URL de la Revista: http://visiondefuturo.fce.unam.edu.ar/index.php/visiondefuturo/index

URL del Documento: https://visiondefuturo.fce.unam.edu.ar/index.php/visiondefuturo/issue/view/18

ISSN 1668 - 8708 - Versión en Línea

E-mail: revistacientifica@fce.unam.edu.ar 
Figura $\mathbf{N}^{\circ}$ 2. (cont.) Formulario de encuesta

6. Cantidad de materias aprobadas (hasta $16 / 06 / 17$ ) *

El valor debe ser un nùmemo.

7. ¿Cuántas veces concurnís a la Facultad por semana?*

El valor dabe ser un númera.

8. Qué medio de transporte es el que más utilizás para ir/venir a la facultad? *

Selveciona la respuesta

9. ¿Desde donde venís? (intersección de calles) *

Escribu su respuesta

10. ¿Cuántos cuadernos usas en un cuatrimestre?*

El valor debe ser un numera.

11. ¿Cuántas hojas imprimis en un cuatrimestre?*

Entre Dy 250

Enare 250 y 500

más de 500

12. ¿Cuántas fotocopias sacas en un cuatrimestre?*

Entre Dy 250

Entre 250 y 500

más de 500

\section{Envis}

Fuente: Elaboración Propia

La Facultad de Ciencias Económicas tiene varias sedes distribuidas en la Ciudad de Buenos Aires y Gran Buenos Aires, en donde los accesos están asegurados por transporte público de diversa índole (ómnibus, tren, subterráneo, etc.). La Facultad no tiene campus con alojamiento, por lo que el alumnado debe trasladarse cada vez que va a clase.

Respecto al papel, el consumo por parte de los estudiantes está en la compra de apuntes que adquieren en la fotocopiadora que se encuentra en la Facultad y cuadernos universitarios. Por lo general, si bien el material de estudio está disponible en forma electrónica, los alumnos suelen utilizarlo en formato papel y acompañar su uso con otros archivos en formato electrónico.

"Visión de Futuro" Año 17, Volumen No 24 N², Julio - Diciembre 2020 - Pág 125 - 145

URL de la Revista: http://visiondefuturo.fce.unam.edu.ar/index.php/visiondefuturo/index

URL del Documento: https://visiondefuturo.fce.unam.edu.ar/index.php/visiondefuturo/issue/view/18

ISSN 1668 - 8708 - Versión en Línea

E-mail: revistacientifica@fce.unam.edu.ar 
A continuación, se describirán las consideraciones realizadas para los dos aspectos considerados: combustible utilizado para la movilidad y papel empleado por los alumnos para estudiar.

\section{Transporte}

Las emisiones de gases de efecto invernadero (GEI) atribuibles a la utilización de combustibles fósiles han demostrado una contribución directa al incremento de la temperatura regional en proporciones mayores a las que se habrían presentado de forma natural. De éstas, las más relevantes que se derivan del sector transporte son: dióxido de carbono $\left(\mathrm{CO}_{2}\right)$; metano $\left(\mathrm{CH}_{4}\right)$ y óxido nitroso $\left(\mathrm{N}_{2} \mathrm{O}\right)$

Para el cálculo de emisiones por tipo de contaminante la ecuación utilizada en el presente trabajo (Tuia et al., 2007) es la siguiente (3):

$$
E_{c}=\sum_{j} N_{j} M_{j} F E_{j c}
$$

Dónde:

EC: emisión del contaminante criterio c en gramos durante la unidad de tiempo elegida (g/tiempo),

$\mathrm{N}_{\mathrm{j}}$ : cantidad de vehículos del tipo j (Número de vehículos),

$M_{\mathrm{j}}$ : kilómetros recorridos por todos los vehículos del tipo j por unidad de tiempo ( $\mathrm{km} / \mathrm{tiempo}$ ),

$F E_{j c}$ : factor de emisión asociado al vehículo del tipo j para el contaminante $c(\mathrm{~g} / \mathrm{km})$.

La fórmula indica que la cantidad total de emisiones de un contaminante determinado es una función de la cantidad de vehículos considerados en el análisis, así como de la distancia total recorrida por cada uno de éstos y de los factores de emisión asociados con cada tipo o categoría de vehículo. En este artículo se trabajará únicamente emisiones de $\mathrm{CO}_{2}$.

Como hemos mencionado, se recolectarán datos relacionados con el tipo de vehículo empleado por los alumnos para trasladarse a la sede universitaria. Los mismos fueron clasificados en: vehículos particulares, motos, colectivo, bicicleta o a pie, subte y tren. Por otro lado, para conocer la distancia recorrida se consultó sobre el origen y destino de esos viajes y si comparten vehículos particulares.

Para procesar los datos recolectados se emplea la siguiente ecuación que es una ampliación de la Ecuación (3):

$$
E_{\mathrm{co} 2}=\sum_{i=1} N \quad V_{i} D_{i} F E_{i C O 2}
$$

Dónde

i: número de encuesta.

$\mathrm{N}$ : número total de encuestas.

$E_{\mathrm{CO} 2}$ : emisión del $\mathrm{CO}_{2}$ en gramos durante una semana de actividad académica ( $\mathrm{g} /$ semana).

$V_{i}$ : número de viajes realizados por el vehículo de la encuesta i en una semana (viajes/semana).

$D_{i}$ : distancia recorrida en kilómetros por el vehículo de la encuesta i en cada viaje ( $\left.\mathrm{km} / \mathrm{viaje}\right)$.

"Visión de Futuro" Año 17, Volumen No 24 N², Julio - Diciembre 2020 - Pág 125 - 145

URL de la Revista: http://visiondefuturo.fce.unam.edu.ar/index.php/visiondefuturo/index

URL del Documento: https://visiondefuturo.fce.unam.edu.ar/index.php/visiondefuturo/issue/view/18

ISSN 1668 - 8708 - Versión en Línea

E-mail: revistacientifica@fce.unam.edu.ar 
FEic: factor de emisión IVE asociado al vehículo de la encuesta i para $\mathrm{CO}_{2}(\mathrm{~g} / \mathrm{km})$.

Cada una de la $\mathrm{N}$ encuestas se procesa por separado, obteniendo un resultado por encuesta para cada una de las variables necesarias en la evaluación de las emisiones totales (EC). El número de viajes por vehículo $\left(V_{i}\right)$ se estima a partir de la pregunta sobre el número de viajes que se hacen en la semana hacia la institución. Los kilómetros recorridos por cada tipo de vehículo $\left(D_{i}\right)$ se estiman mediante un sistema de información geográfica de acceso libre (Google Earth), haciendo una consulta de distancias entre el punto de partida de los viajes y la institución. Los factores de emisión por contaminante para los tipos de vehículo particular y motocicletas $\left(\mathrm{FE}_{\mathrm{ic}}\right)$ se toman de acuerdo a cada uno de los vehículos por encuesta.

Los factores de emisión $\mathrm{CO}_{2}$ empleados en este estudio se expresan en la Tabla 1.

Tabla $\mathrm{N}^{\circ}$ 1. Factores de emisión de $\mathrm{CO} 2$

\begin{tabular}{|c|c|c|c|c|}
\hline Pasajeros & $\begin{array}{c}\text { Factor de Emisión } \\
\mathrm{CO}_{2}\end{array}$ & unidades & $\begin{array}{c}\text { Consumo } \\
\text { combustible }\end{array}$ & \\
\hline Auto nafta & 0,196 & $\mathrm{~kg} /$ vehkm & & \\
\hline Bus diésel & 1,197 & $\mathrm{~kg} / \mathrm{vehkm}$ & & \\
\hline Ferrocarril diésel & 2,690 & $\mathrm{~kg} /$ litro & 4,84 & litros/trenkm \\
\hline Ferrocarril eléctrico & 0,44 & $\mathrm{~kg} / \mathrm{KWH}$ & 3,54 & $\mathrm{KWH} /$ trenkm \\
\hline
\end{tabular}

Fuente: Datos relevados del Observatorio de Movilidad Urbana (OMU)

Se asume que $100 \%$ de los vehículos del tipo auto utilizan nafta como combustible, debido a que aquellos que utilizan diésel no son significativos. El inventario 2012 de Energía en la categoría transporte no computa automóviles de pasajeros con diésel.

Una vez obtenidas las emisiones de $\mathrm{CO}_{2}$ y conociendo factor de fijación media se calcula la Huella Ecológica movilidad per cápita, anteriormente enunciada en (1).

\section{Papel}

Para calcular la HE papel de los estudiantes de Análisis Matemático II, en primer lugar, relevamos a través de la encuesta el consumo por cuatrimestre de papel de los estudiantes. Se les consultó por las tres categorías preponderantes de consumo de papel en las actividades universitarias: cantidad de cuadernos que usan para estudiar, fotocopias que realizan y papel que imprimen.

En lo que se refiere a los cuadernos el más utilizado por los alumnos es el que presenta las siguientes características: con espiral de tapa blanda, contiene 84 hojas de $65 \mathrm{gr} / \mathrm{m} 2$ y 223 × $295 \mathrm{~mm}$ de tamaño. Con respecto a las hojas para otros usos, tanto en la fotocopiadora como para impresión hogareña, las mismas tienen un gramaje de $75 \mathrm{gr} / \mathrm{m} 2$ y sus medidas son 210 x 297 mm. (A4).

\footnotetext{
"Visión de Futuro" Año 17, Volumen N²4 N², Julio - Diciembre 2020 - Pág 125 - 145

URL de la Revista: http://visiondefuturo.fce.unam.edu.ar/index.php/visiondefuturo/index

URL del Documento: https://visiondefuturo.fce.unam.edu.ar/index.php/visiondefuturo/issue/view/18

ISSN 1668 - 8708 - Versión en Línea

E-mail: revistacientifica@fce.unam.edu.ar
} 
Para determinar la Huella Ecológica parcial por consumo de papel se utiliza (2). Para realizar dicho cálculo, es necesario conocer el consumo de papel en toneladas. Para ello, se plantean dos fórmulas que convierten la cantidad de cuadernos y de hojas utilizadas por los alumnos en toneladas de papel. Para realizar esta conversión se utilizan los siguientes factores de conversión analizados en Fernandez et al., 2017:

$$
\begin{aligned}
& \text { Tonelada de papel por cada cuaderno utilizado }=\frac{0,065\left(\mathrm{~kg} / \mathrm{m}^{2}\right) * 84 \text { hojas }}{15,15\left(\mathrm{hojas} / \mathrm{m}^{2}\right) * 1000(\mathrm{~kg} / \mathrm{tn})}
\end{aligned}
$$

$$
\text { Tonelada de papel por cada cuaderno utilizado }=0,00036 \text { (tn/cuaderno) }
$$

$$
\begin{gathered}
\text { Tonelada de papel por cada hoja utilizada }=\frac{0,08\left(\mathrm{~kg} / \mathrm{m}^{2}\right)}{16,033\left(\text { hojas } / \mathrm{m}^{2}\right) * 1000(\mathrm{~kg} / \mathrm{tn})}
\end{gathered}
$$

Tonelada de papel por cada hoja utilizada $=0,000005$ (tn/hoja)

Por otro lado, para determinar la productividad se supuso que en promedio para producir 1 tonelada de papel se necesitan $2 \mathrm{~m}^{3}$ de madera (Guerrero y Guiñirgo, 2008). La productividad ecológica forestal promedio que se va a utilizar es la propuesta por Wackernagel y Rees (2001), que es de 2,3 $\mathrm{m}^{3}$ de fibra maderera utilizable por hectárea por año, es decir que la productividad será:

$$
\begin{gathered}
\text { Productividad anual (tonelada/ha) } \\
2\left(\mathrm{~m}^{3} / \mathrm{tn}\right)
\end{gathered}
$$

La productividad nos indica cuantas toneladas de fibra de celulosa podemos obtener en una hectárea de tierra forestal. En el siguiente apartado utilizaremos estos factores para calcular la HE papel, utilizando la ecuación (2).

\section{Resultados}

\section{Características de la población}

El estudio se centró en cuatro cursos de la asignatura Análisis Matemático II. Dicha materia se dicta en el primer y segundo cuatrimestre para las carreras de Licenciado en Economía, Actuario en Economía y Actuario en Administración en la Facultad de Ciencias Económicas de la Universidad de Buenos Aires. En total hubo 375 alumnos inscriptos en ese cuatrimestre en las comisiones relevadas.

Los siguientes datos analizados reúnen las respuestas obtenidas a partir del cuestionario online administrado a 109 alumnos. En promedio, cada encuestado demoró 3 minutos y 23 segundos en completar la encuesta.

La ficha técnica de la encuesta es la siguiente:

"Visión de Futuro" Año 17, Volumen N²4 N², Julio - Diciembre 2020 - Pág 125 - 145

URL de la Revista: http://visiondefuturo.fce.unam.edu.ar/index.php/visiondefuturo/index

URL del Documento: https://visiondefuturo.fce.unam.edu.ar/index.php/visiondefuturo/issue/view/18

ISSN 1668 - 8708 - Versión en Línea

E-mail: revistacientifica@fce.unam.edu.ar 
Fecha de realización de las encuestas: 20 de marzo del 2017 al 23 de junio del 2017

Instrumento de recolección de datos: Cuestionario online autoadministrado

Cantidad de encuestas realizadas: 109 casos.

Marco muestral: alumnos de Análisis Matemático II de los cuatro cursos involucrados en la encuesta.

Diseño muestral: Muestreo no probabilístico. Autoselección

Cursos de participaron: tres cursos de la sede Paternal de los horarios: 9 a 11hs., 17 a $19 \mathrm{hs}$. y 19 a 21 hs. y un curso de la sede Córdoba de 7 a 9hs.

Respecto a la carrera que estudian, el 66\% son estudiantes de la Carrera de Lic. En Economía, el $17 \%$ de Actuario en Economía y el 17\% de Actuario en Administración.

Del total de encuestados, el 68\% asiste a la Sede Paternal y el 32\% a la Sede Córdoba. Ambas están en la Ciudad de Buenos Aires, en los Barrios de La Paternal y Balvanera respectivamente.

El $57 \%$ de los alumnos encuestados trabajan y tienen aprobados en promedio 9 materias de un total de 34.

Un $63 \%$ de los alumnos cursan por la mañana (7 a 13hs.), y el resto por la tarde (13 a 19hs.) y noche (19 a 23hs.).

\section{Resultados sobre el aspecto movilidad}

De los 109 alumnos encuestados en el Gráfico 1 se observa que el mayor porcentaje de la población estudiantil utiliza el Transporte Público para dirigirse a la Universidad (85\%). En segundo lugar, con porcentaje mucho menor, se emplea el Transporte Personal (11\%) y por último un $4 \%$ concurre caminando o en bicicleta.

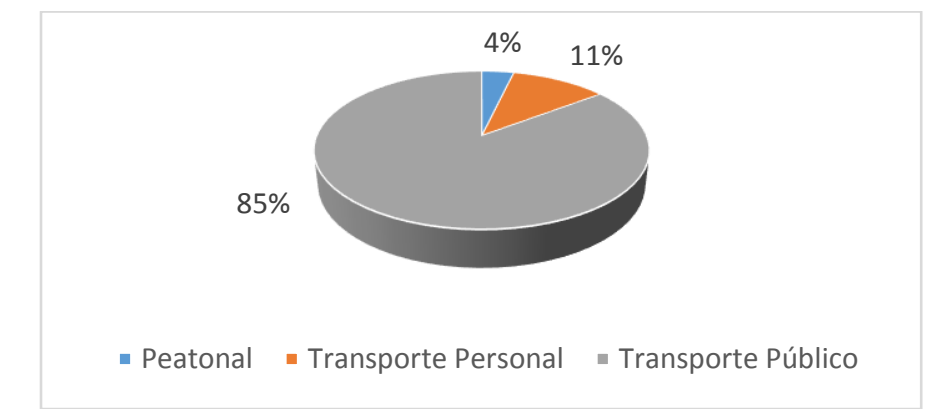

\section{Gráfico $N^{\circ}$ 1. Tipo de transporte empleado por el alumno}

Fuente: total de casos (109 encuestados)

Como los estudiantes asisten no solo desde diversos puntos de la Ciudad de Buenos Aires, sino también desde localidades del conurbano bonaerense, resulta acorde que el porcentaje de alumnos a pie o en bicicleta sea tan bajo. Además, las sedes de cursada se encuentran bien comunicadas con diversos tipos de transporte público, resultando más económico asistir por este medio.

\footnotetext{
"Visión de Futuro" Año 17, Volumen No 24 Nº 2, Julio - Diciembre 2020 - Pág 125 - 145

URL de la Revista: http://visiondefuturo.fce.unam.edu.ar/index.php/visiondefuturo/index

URL del Documento: https://visiondefuturo.fce.unam.edu.ar/index.php/visiondefuturo/issue/view/18

ISSN 1668 - 8708 - Versión en Línea

E-mail: revistacientifica@fce.unam.edu.ar
} 
En la tabla 2 y gráfico 2 se detalla con mayor especificidad que tipo de transporte público utiliza el estudiante encuestado.

Tabla $\mathbf{N}^{\circ}$ 2. Medio de transporte utilizado

\begin{tabular}{|c|c|c|c|c|c|}
\hline A pie/Bicicleta & Auto & Colectivo & Subte & Tren & $\begin{array}{c}\text { Combinación de } \\
\text { Transporte Público }\end{array}$ \\
\hline 4 & 12 & 66 & 8 & 3 & 16 \\
\hline
\end{tabular}

Fuente: total de casos (109 encuestados)

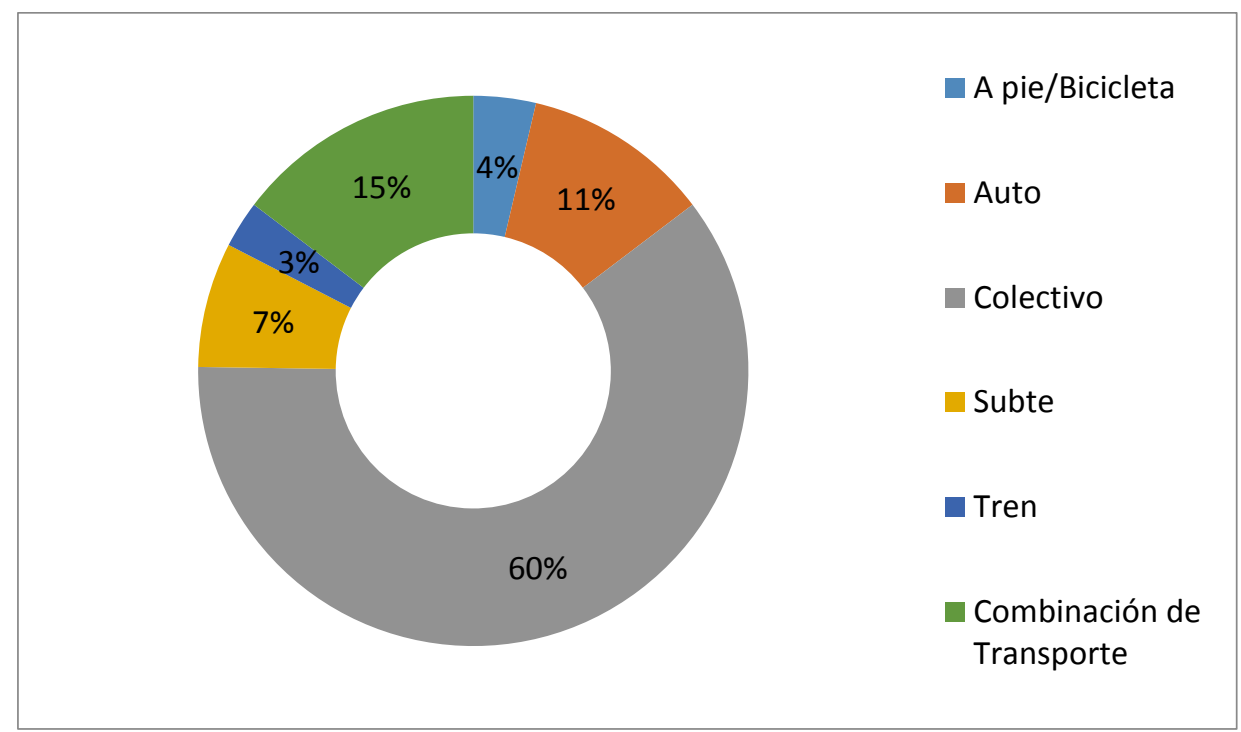

Gráfico $\mathbf{N}^{\circ}$ 2. Medio de transporte utilizado

Fuente: total de casos (109 encuestados)

Una de las sedes no tiene acceso por medio del subterráneo, por lo que el transporte en colectivo resulta el más usado.

Para procesar la información obrante en Tabla 3, se consideraron las capacidades de los medios de transporte, considerando que todos los pasajeros van sentados. Para el caso de los trenes, esto difiere de acuerdo a la Línea (Mitre-Roca-San Martín y Urquiza). Los datos que se emplean provienen de base de datos de FA (Ferrocarriles Argentinos). Para el caso de los subtes se consideró en este trabajo el coche marca Fiat que tiene capacidad de 41 pasajeros sentados, 129 parados, es decir 170 pasajeros a plena carga. Para una formación de 5 vagones su carga máxima es de 850 personas (SBASE - Subterráneos de Bs. As.)

Los colectivos de la Ciudad de Buenos Aires poseen en general una capacidad de 33 pasajeros sentados y 50 pasajeros con carga máxima (CEAP - Cámara Empresaria de Autotransporte de Pasajeros). Para los autos, la capacidad mínima es 1 y capacidad máxima fue considerada de 4 pasajeros.

El factor de emisión de $\mathrm{CO}_{2}$, como ya hemos mencionado, tiene relación directa con el tipo de transporte y la cantidad de pasajeros que comparten el modo.

"Visión de Futuro" Año 17, Volumen No 24 N², Julio - Diciembre 2020 - Pág 125 - 145

URL de la Revista: http://visiondefuturo.fce.unam.edu.ar/index.php/visiondefuturo/index

URL del Documento: https://visiondefuturo.fce.unam.edu.ar/index.php/visiondefuturo/issue/view/18

ISSN 1668 - 8708 - Versión en Línea

E-mail: revistacientifica@fce.unam.edu.ar 
Así, en la siguiente tabla se muestran las variaciones del factor de emisión por km y por persona para los diferentes modos de transporte y los diferentes escenarios de capacidad que se pudiesen presentar.

Tabla $N^{\circ}$ 3. Factores de emisión

\begin{tabular}{|c|c|c|c|c|c|c|c|}
\hline & \multicolumn{4}{|c|}{ Tren } & \multirow[t]{2}{*}{ Subte } & \multirow[t]{2}{*}{ Colectivo } & \multirow[t]{2}{*}{ Auto } \\
\hline & Mitre & Roca & $\begin{array}{l}\text { San } \\
\text { Martín }\end{array}$ & Urquiza & & & \\
\hline Cap. Mínima (sentados) & 336 & 288 & 449 & 272 & 205 & 33 & 1 \\
\hline $\begin{array}{c}\text { Cap. Máxima } \\
\text { (sentados y parados) }\end{array}$ & 1094 & 1046 & 1307 & 896 & 850 & 50 & 4 \\
\hline $\mathrm{FC} \mathrm{CO}_{2}(\mathrm{~kg} / \mathrm{km})$ & 1,55 & 1,55 & 13,02 & 1,55 & 1,55 & 1,1970 & 0,196 \\
\hline $\begin{array}{c}\mathrm{FC} \mathrm{CO} 2(\mathrm{~kg} / \mathrm{km} / \mathrm{pers}) \\
\text { carga mínima }\end{array}$ & 0,005 & 0,005 & 0,029 & 0,006 & 0,008 & 0,036 & 0,196 \\
\hline $\begin{array}{c}\mathrm{FC} \mathrm{CO}(\mathrm{kg} / \mathrm{km} / \text { pers. }) \\
\text { carga máxima }\end{array}$ & 0,001 & 0,001 & 0,010 & 0,002 & 0.002 & 0,024 & 0,049 \\
\hline Diferencia & 0,003 & 0,004 & 0,019 & 0,004 & 0,006 & 0,012 & 0,147 \\
\hline $\begin{array}{c}\text { Porcentaje de } \\
\text { variación FC CO} \\
(\mathrm{kg} / \mathrm{km} / \text { pers })\end{array}$ & $60 \%$ & $80 \%$ & $65 \%$ & $67 \%$ & $75 \%$ & $33 \%$ & $75 \%$ \\
\hline
\end{tabular}

Fuente: Elaboración Propia en base a datos FA-CEAP-SBASE

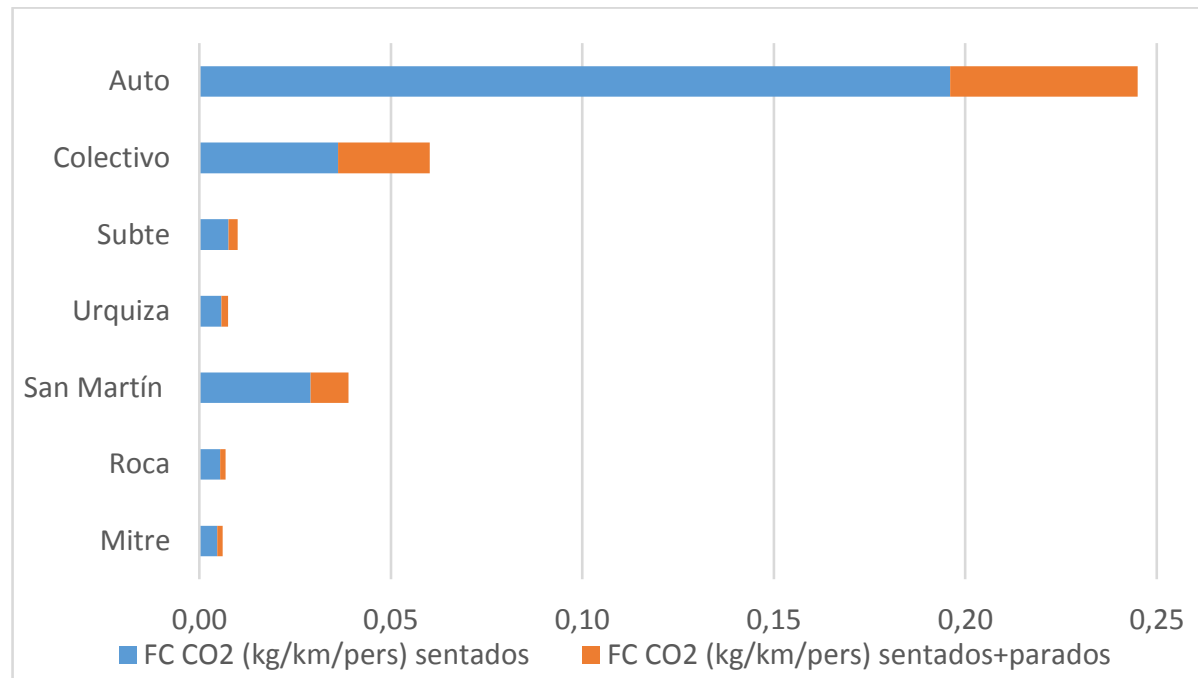

Gráfico $\mathrm{N}^{\circ}$ 3. Factor de Emisión $\mathrm{CO}_{2}(\mathrm{~kg} / \mathrm{km} /$ pers.)

Fuente: Elaboración propia en base a tabla 3

\section{Emisión de $\mathrm{CO}_{2}$}

Para el cálculo de la emisión de $\mathrm{CO}_{2}\left(\mathrm{E}_{\mathrm{CO} 2}\right)$ debido a la movilidad de los estudiantes encuestados se consideró la fórmula (4) enunciada en la Sección 2.3.1.

Se calcularon las emisiones de $\mathrm{CO}_{2}$ semanales para los estudiantes encuestados en relación a la cantidad de km. recorridos desde sus hogares hacia las sedes universitarias, considerando la 
cantidad de viajes semanales que se efectúan semanalmente y el factor de emisión asociado al transporte utilizado. Se tuvo en cuenta que algunos estudiantes combinan dos tipos de transporte y

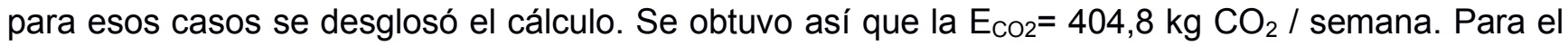
cálculo de la emisión de $\mathrm{CO}_{2}$ anual se consideró que cada cuatrimestre tiene una duración de 12 semanas, totalizando 32 semanas en el año. Por lo tanto, para el año 2018 donde se efectivizó la encuesta de 109 alumnos de las Sedes Paternal y Córdoba de la FCE - UBA, se totalizó una emisión de ese contaminante de: $\mathrm{E}_{\mathrm{CO}_{2}}=12953.54 \mathrm{~kg} \mathrm{CO} /$ año. Por lo tanto, cada estudiante emitió, en promedio, 118,84 kg/individuo/año.

Para realizar una comparación entre el Dióxido de Carbono emitido por 400 alumnos en relación a las emisiones de ciertos sectores industriales, como por ejemplo la Industria del Acero que en promedio emite 0,267 tn $\mathrm{CO}_{2} /$ tn producto, la emisión de $\mathrm{CO}_{2}$ anual de los 400 estudiantes para desplazarse a las sedes universitarias anualmente sería el equivalente al dióxido de carbono producido para fabricar 118 tn de acero. Para el caso de la Industria del Cemento, que en promedio emite 0,745 tn $\mathrm{CO}_{2}$ / th producto, la emisión de $\mathrm{CO}_{2}$ producido por los estudiantes sería equivalente a la producción de 63 tn de Cemento.

En la Tabla 4, se realiza una comparación de emisiones de $\mathrm{CO}_{2}$, teniendo en cuenta los diferentes medios de transporte y la población estudiada. Del análisis de los datos relevados, se obtiene que los alumnos concurren en promedio 4 veces por semana para cursar asignaturas y la distancia recorrida media es de $11,34 \mathrm{~km}$.

En general los alumnos se desplazan en horas pico hacia las sedes universitarias, se supone que la carga del transporte público es máxima. A nivel ciudad la emisión de dióxido carbono es superior, ya que aumenta la cantidad de vehículos que circulan (mayor frecuencia de transporte público y mayor cantidad de vehículos privados).

De dicha comparación se observa la gran diferencia existente entre el uso de auto y el colectivo y también la gran diferencia entre la emisión del colectivo y el subte. Obviamente, esto se debe a la diferencia entre los factores de emisión y también el hecho de la capacidad de carga de cada vehículo.

Tabla $\mathrm{N}^{\circ}$ 4. Emisiones $\mathrm{CO}_{2}$ (kg/km/pers.) en relación a la capacidad de carga

\begin{tabular}{|c|c|c|c|c|}
\hline \multirow[t]{2}{*}{ Medio de Transporte } & \multicolumn{2}{|c|}{ Carga Mínima (kg/km/pers) } & \multicolumn{2}{|c|}{ Carga Completa (kg/km/pers) } \\
\hline & Semanal & Emisión Anual & Semanal & Emisión Anual \\
\hline \multirow{5}{*}{$\begin{array}{c}\text { Auto } \\
\text { Bus diésel } \\
\text { Ferrocarril diésel } \\
\text { Ferrocarril eléctrico } \\
\text { Subte }\end{array}$} & 7112 & 227598 & 1778 & 56899 \\
\hline & 1316 & 42120 & 26 & 842 \\
\hline & 1053 & 33687 & 0,8 & 26 \\
\hline & 168 & 5383 & 0,1 & 5 \\
\hline & 276 & 8823 & 0,4 & 14 \\
\hline
\end{tabular}

Fuente: Elaboración Propia

\footnotetext{
"Visión de Futuro" Año 17, Volumen N²4 N², Julio - Diciembre 2020 - Pág 125 - 145

URL de la Revista: http://visiondefuturo.fce.unam.edu.ar/index.php/visiondefuturo/index

URL del Documento: https://visiondefuturo.fce.unam.edu.ar/index.php/visiondefuturo/issue/view/18

ISSN 1668 - 8708 - Versión en Línea

E-mail: revistacientifica@fce.unam.edu.ar
} 


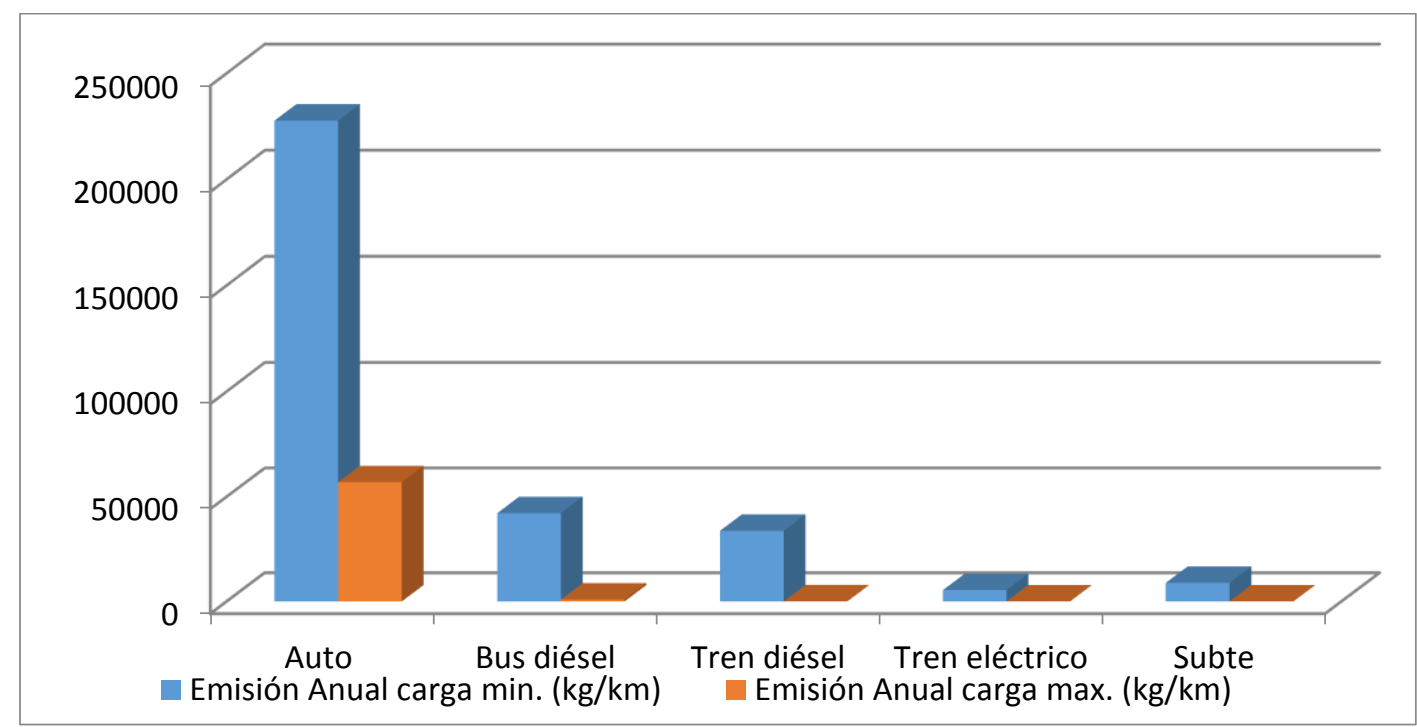

Gráfico $\mathrm{N}^{\circ}$ 4. Comparación Emisiones Anual $\mathrm{CO}_{2}$ para carga mínima y máxima Fuente: Elaboración Propia

\section{Huella ecológica movilidad}

Finalmente se calcula el Indicador de la Huella Ecológica movilidad per cápita, que estudia el impacto del uso de los medios de transporte para el desplazamiento de los estudiantes a las sedes universitarias en término de superficies productivas de los ecosistemas.

Este indicador se calcula utilizando la fórmula (1). La fijación media de carbono para un terreno de tipo forestal se estima en 1,8 tnC/ha/año (Wackernagel y Rees, 2001), que traducido a fijación de $\mathrm{CO}_{2}$ equivale a 6,6 tn $\mathrm{CO}_{2} / \mathrm{ha} / a n ̃ o$. Realizando el cálculo correspondiente para 400 alumnos se obtiene una Huella Ecológica de 7,2 ha/año. Por lo tanto, se necesitan 7,2 ha de terreno de tipo forestal para asimilar el dióxido de carbono emitido por los 400 estudiantes de Análisis Matemático II por el término de un año.

\section{Resultados sobre el consumo de papel}

El papel es el principal insumo que utilizan los estudiantes para desarrollar sus actividades académicas, ya sea para tomar notas como en el uso de fotocopias y apuntes. Para calcular su consumo, como ya hemos mencionado, consultamos a los alumnos sobre el uso de cuadernos universitarios, hojas impresas y fotocopiadas. Se dejó fuera del análisis de este estudio la compra de libros impresos.

En lo que se refiere a los cuadernos utilizados por los alumnos en un cuatrimestre, se han obtenido los siguientes resultados:

\footnotetext{
"Visión de Futuro" Año 17, Volumen No 24 N², Julio - Diciembre 2020 - Pág 125 - 145

URL de la Revista: http://visiondefuturo.fce.unam.edu.ar/index.php/visiondefuturo/index

URL del Documento: https://visiondefuturo.fce.unam.edu.ar/index.php/visiondefuturo/issue/view/18

ISSN 1668 - 8708 - Versión en Línea

E-mail: revistacientifica@fce.unam.edu.ar
} 
Tabla $N^{\circ} 5$. Uso de cuadernos en un cuatrimestre

\begin{tabular}{|c|c|}
\hline Cantidad de cuadernos & $\begin{array}{c}\text { Cantidad de } \\
\text { alumnos }\end{array}$ \\
\hline 2 & 22 \\
\hline 3 & 40 \\
\hline 4 & 26 \\
\hline 5 & 11 \\
\hline 6 & 5 \\
\hline mayor a 6 & 5 \\
\hline
\end{tabular}

Fuente: Base total de encuestados (109 casos)

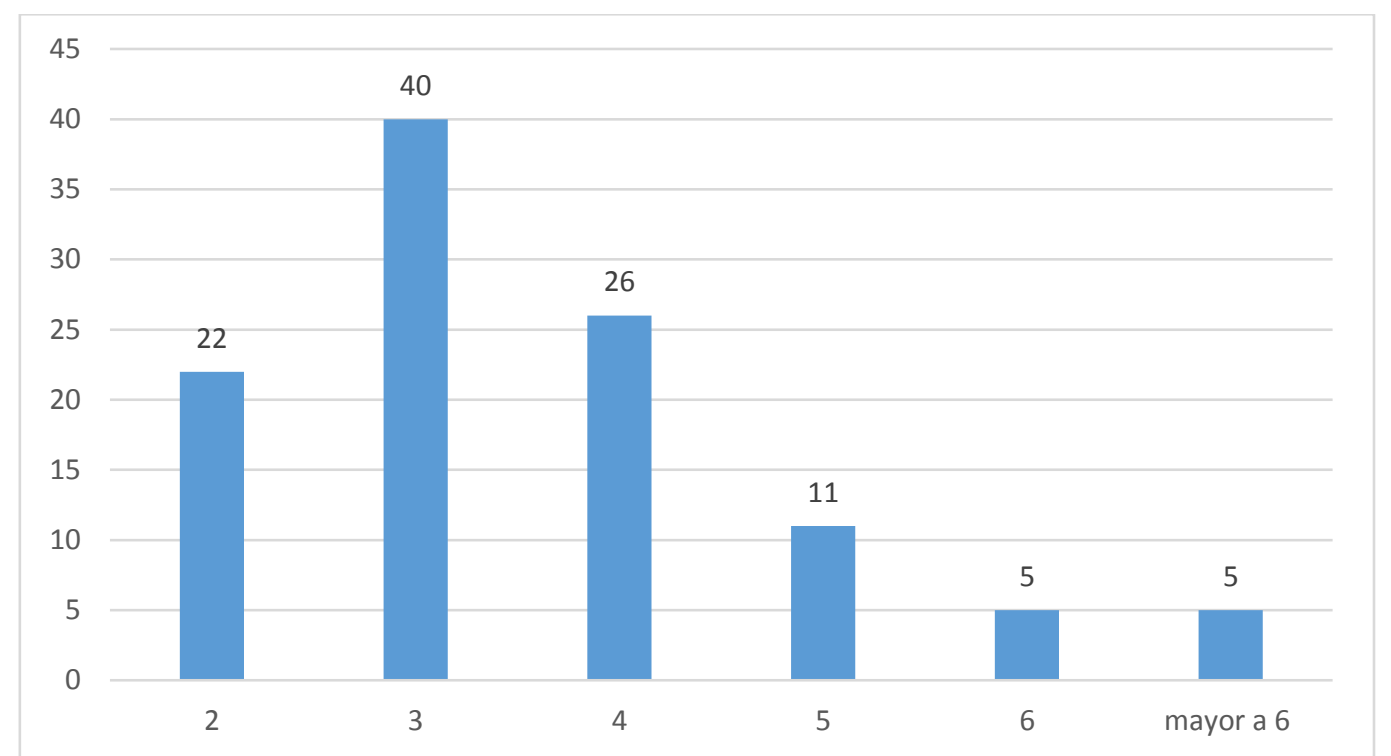

Gráfico $\mathrm{N}^{\circ}$ 5. Cantidad de cuadernos utilizados por los alumnos encuestados en un cuatrimestre Fuente: Base total de encuestados (109 casos)

Se observa que los 109 alumnos encuestados utilizan 391 cuadernos en un cuatrimestre, si consideramos que los alumnos en un año cursan dos cuatrimestres, utilizan 782 cuadernos en el año. Por lo tanto, la media de consumo es de $7,17 \cong 7$ cuadernos por alumno al año.

Con respecto a los cuadernos utilizados, consideramos que, si un alumno en promedio utiliza 7 cuadernos universitarios por año, en toneladas de papel representan:

Tonelada de papel por cada cuaderno utilizado=0,00036 (tn/cuaderno)

Toneladas de papel por el consumo de cuaderno de un alumno $=0,00036$ * 7

Toneladas de papel por el consumo de cuadernos de un alumno en un año $=0,0025$

Veamos ahora que sucede con el consumo de papel en las fotocopiadores y al imprimir. En lo que se refiere al consumo cuatrimestral de hojas impresas por los alumnos, el $71 \%$ indicó que utiliza entre 0 y 250 hojas impresas, $23 \%$ entre 250 y 500 hojas impresas y sólo un $6 \%$ utiliza más de 500 hojas impresas.

"Visión de Futuro" Año 17, Volumen No 24 N², Julio - Diciembre 2020 - Pág 125 - 145

URL de la Revista: http://visiondefuturo.fce.unam.edu.ar/index.php/visiondefuturo/index

URL del Documento: https://visiondefuturo.fce.unam.edu.ar/index.php/visiondefuturo/issue/view/18

ISSN 1668 - 8708 - Versión en Línea

E-mail: $\underline{\text { revistacientifica@fce.unam.edu.ar }}$ 


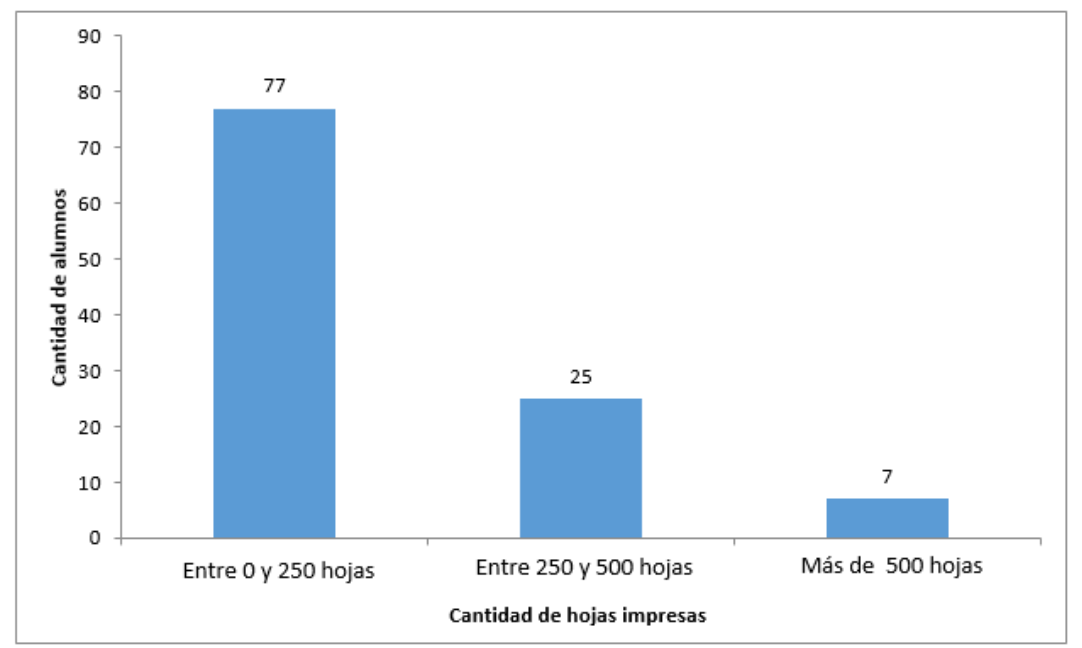

Gráfico $\mathrm{N}^{\circ}$ 6. Hojas impresas por los alumnos en un cuatrimestre Fuente: Base total de encuestados (109 casos)

Con respecto al uso de fotocopias, los alumnos encuestados indicaron que en un $43 \%$ utilizan entre 250 y 500 hojas, un $29 \%$ más de 500 hojas fotocopiadas y un $28 \%$ entre 0 y 250 hojas fotocopiadas.

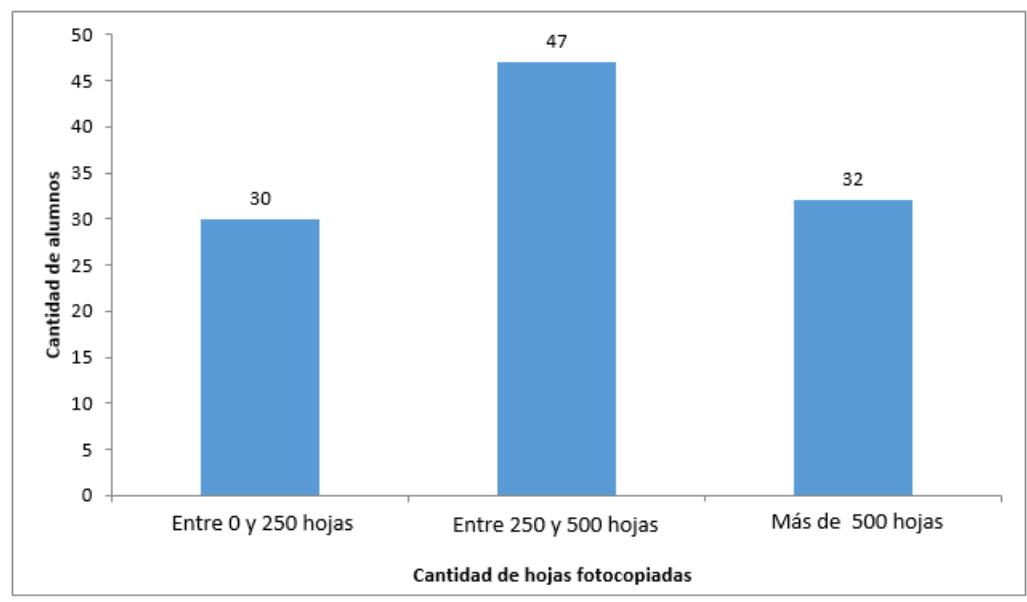

Gráfico $N^{\circ} 7$. Hojas fotocopiadas por los alumnos por cuatrimestre

Fuente: Base total de encuestados (109 casos)

Comparando el gráfico de hojas impresas y de hojas fotocopiadas, se observa que los alumnos que respondieron la encuesta fotocopian más que lo que imprimen en impresoras personales. Por lo tanto, los esfuerzos para disminuir el impacto ambiental de consumo de papel deben estar puestos en que se fotocopie sólo lo necesario.

Para realizar el cálculo de Huella Ecológica del papel debido al consumo de hojas impresas y fotocopiadas agrupamos ambos consumos cuatrimestrales, de forma de calcular el consumo anual de hojas por alumno. Si juntamos ambos consumos se observa la tabla 8.

"Visión de Futuro" Año 17, Volumen No 24 N² 2, Julio - Diciembre 2020 - Pág 125 - 145

URL de la Revista: http://visiondefuturo.fce.unam.edu.ar/index.php/visiondefuturo/index

URL del Documento: https://visiondefuturo.fce.unam.edu.ar/index.php/visiondefuturo/issue/view/18

ISSN 1668 - 8708 - Versión en Línea

E-mail: revistacientifica@fce.unam.edu.ar 
Tabla $N^{\circ}$ 6. Consumo cuatrimestral de hojas tamaño A4

\begin{tabular}{|c|c|c|c|}
\hline \multirow{2}{*}{$\begin{array}{c}\text { Tipo de uso del } \\
\text { papel }\end{array}$} & \multicolumn{3}{|c|}{ Consumo cuatrimestral de hojas tamaño A4 } \\
\cline { 2 - 4 } & 0 a 250 & 250 a 500 & Más de 500 \\
\hline Impreso & 77 & 25 & 7 \\
\hline Fotocopia & 30 & 47 & 32 \\
\hline
\end{tabular}

Fuente: Base total de encuestados (109 casos)

De esta forma se calcula en base a los resultados de la encuesta los siguientes consumos promedios de papel por alumno: 222 hojas de papel impreso en un cuatrimestre, por lo que anualmente consideramos que consume el doble (dos cuatrimestres): 444 hojas A4 impresas anualmente. Con respecto a las hojas fotocopiadas por alumno en un cuatrimestre en promedio: 416 hojas fotocopiadas en un cuatrimestre, por lo que anualmente consideramos que consume el doble (dos cuatrimestres): 832 hojas A4 fotocopiadas en un año.

Tabla $N^{\circ}$ 7. Consumo anual promedio de hojas A4

\begin{tabular}{|c|c|}
\hline Tipo de uso del papel & $\begin{array}{c}\text { Consumo anual en promedio } \\
\text { por alumno de hojas A4 }\end{array}$ \\
\hline Impreso & 444 \\
\hline Fotocopia & 832 \\
\hline Total & 1276 \\
\hline
\end{tabular}

Fuente: Base total de encuestados (109 casos)

Ahora debemos calcular cuantas toneladas pesan las 1276 hojas consumidas. Para esto, usamos el factor definido anteriormente:

Tonelada de papel por cada hoja utilizada $=0,000005$ (tn / hojas)

Toneladas anuales de papel consumidas para fotocopiar e imprimir por alumno en promedio $=$ 0,00638

De esta forma las toneladas anuales de papel utilizadas por un alumno son 0,0088 .

Utilizando el factor de productividad anual:

Productividad anual (tn / ha) $=1,15 \mathrm{tn} / \mathrm{ha}$

Hectáreas anuales debido al consumo de papel de un alumno $=0,01$

Es decir, cada alumno consume un equivalente de papel convertido en área de 0,01 Ha (100 m2). Si bien el resultado obtenido no da mucha información al ser analizado en forma aislada, resulta útil para analizar los tres tipos de consumo que tienen los alumnos en papel. El mayor consumo se da por el uso de fotocopias, por lo que el eje estratégico para reducir su consumo debe estar orientado a este tipo.

\footnotetext{
"Visión de Futuro" Año 17, Volumen No 24 N² 2, Julio - Diciembre 2020 - Pág 125 - 145

URL de la Revista: http://visiondefuturo.fce.unam.edu.ar/index.php/visiondefuturo/index

URL del Documento: https://visiondefuturo.fce.unam.edu.ar/index.php/visiondefuturo/issue/view/18

ISSN 1668 - 8708 - Versión en Línea

E-mail: revistacientifica@fce.unam.edu.ar
} 


\section{CONCLUSION}

En la Ciudad de Buenos Aires el transporte púbico de mayor envergadura es el colectivo ya que tiene muchas líneas y tiene una gran diversidad de recorridos. Esto se manifiesta en los resultados de la encuesta realizada, donde se aprecia que un $66 \%$ de los estudiantes utilizan este medio. Sólo el $4 \%$ elige medios no contaminantes (a pie o bicicleta).

En primer lugar realizamos un análisis desde el punto de vista de políticas públicas con relación a las emisiones de $\mathrm{CO} 2$ provocadas por el empleo del transporte público y privado y enunciaremos posibles estrategias para reducir las emisiones de GEI basado en el Plan de Acción Buenos Aires 2030 (Lockhart y Gómez, 2009) cuyo objetivo fue tomar medidas y prepararse para los efectos del calentamiento global, orientada a la planificación de las políticas integrales de mitigación y adaptación a los efectos adversos del cambio climático para el período 2010-2030. Según el diagnóstico realizado, la situación actual en materia de transporte, se caracteriza por una gran cantidad de automóviles particulares y una marcada insuficiencia de oferta de transporte público de pasajeros.

Considerando el crecimiento poblacional al 2030 se estima que habitarán en la Ciudad de Buenos Aires alrededor de 3.198.366 habitantes, generando una mayor demanda / consumo del sector transporte. Debido a que la oferta de transporte público en la Ciudad es insuficiente para atender la demanda generada, resulta prioritaria su ampliación para aumentar la capacidad de acogida del sector, a través de políticas públicas de promoción de medios de transporte masivo y el desaliento de la utilización del automóvil particular, que es el medio más nocivo en cuanto a emisión de GEl. En este sentido, se estima que el fortalecimiento de la red de trenes evitaría el ingreso de 250.000 vehículos aproximadamente.

El uso de la bicicleta es el transporte más adecuado porque no emite factores contaminantes. Ahora, ¿sería entonces mejor ir en bicicleta a todos lados para evitar las emisiones? Sí, sería mejor, pero tampoco es que nos libremos de la huella de carbono. Si los mismos 8 kilómetros que estuvimos calculando para el coche, tren y colectivo, los hiciésemos en una bicicleta la huella de carbono la tendríamos que calcular dependiendo de lo que hayamos comido, ya que ese sería el combustible. Uno quema más o menos 50 calorías por cada kilómetro y medio de pedaleada. Obviamente el uso de la misma o la caminata se tornan en los medios más saludables para las personas que usan estos medios y para el resto de la población por la no emisión de contaminantes.

Todas estas propuestas deben propiciarse a través de la Subsecretaria de Proyectos de Urbanismo, Arquitectura e Infraestructura del Ministerio de Desarrollo Urbano. Sin embargo, impulsar un cambio modal en el transporte requiere no solamente de una mejora sustancial en el transporte

\footnotetext{
"Visión de Futuro" Año 17, Volumen No 24 N², Julio - Diciembre 2020 - Pág 125 - 145

URL de la Revista: http://visiondefuturo.fce.unam.edu.ar/index.php/visiondefuturo/index

URL del Documento: https://visiondefuturo.fce.unam.edu.ar/index.php/visiondefuturo/issue/view/18

ISSN 1668 - 8708 - Versión en Línea

E-mail: revistacientifica@fce.unam.edu.ar
} 
público de pasajeros, sino también conlleva la toma de conciencia por parte de la ciudadanía acerca de los impactos ambientales de los diferentes medios de transporte.

Desde ese lugar, la Universidad como todas las instituciones educativas deben propiciar medidas de prevención en materia de cuidado del medio ambiente y en especial un uso consciente del transporte. Por ejemplo, favorecer el acceso con transporte público a las sedes universitarias e incentivar el uso de la bicicleta como medio de transporte para acceder a las mismas. Esto debe estar acompañado por políticas públicas que mejoren y prioricen el tráfico peatonal, de ciclistas y del transporte público de pasajeros.

En lo que se refiere al consumo de papel por parte de los alumnos encuestados es interesante destacar que lo más utilizan son las fotocopias por lo que el eje estratégico para reducir su consumo debe estar orientado a reducir su uso, utilizando material digitalizado y si es necesario el empleo de papel que sea reciclado.

El beneficio ambiental al consumir menor papel o utilizar papel reciclado son conservar los recursos utilizados para fabricarlo (celulosa, agua y energía) y disminuir el residuo generado al desecharlo.

Por lo tanto, para disminuir el consumo de papel es clave tener todo el material digitalizado, por otro lado, si se desea imprimir se debe hacer en forma eficiente, es decir, a doble faz, con el tamaño de letra legible más pequeño posible y revisar los márgenes de impresión.

De esta forma y en línea con estas recomendaciones de menor consumo de papel una acción que se ha implementado desde la cátedra de Análisis Matemático II es facilitarles a los alumnos las guías y apuntes de la cátedra en el campus virtual.

\section{REFERENCIAS}

Area, M. C., Mastrantonio, G., Vélez, H. (2012). “Gestión ambiental en la fabricación de papel reciclado" en Zanuttini, M. (ed.) Reciclado Celulósico, pp. 264-303. Santa Fe.

Area M.C., Villalba L.L. (1996). "Metodología para el estudio de la capacidad de generación y recolección de papel de desperdicio urbano". Información Tecnológica, 7 (4): 157-164.

Cerutti, J. (2013). Análisis de diagnóstico tecnológico sectorial: celulosa y papel. Ministerio de Ciencia, Tecnología e Innovación Productiva, Buenos Aires.

Fernandez, M. J.; García Fronti, V.; Parma, A. (2017). "Indicador de sostenibilidad ambiental en la Facultad de Ciencias Económicas de la Universidad de Buenos Aires". Gestión Universitaria vol. $10 n^{\circ} 3$.

"Visión de Futuro" Año 17, Volumen No 24 N², Julio - Diciembre 2020 - Pág 125 - 145

URL de la Revista: http://visiondefuturo.fce.unam.edu.ar/index.php/visiondefuturo/index

URL del Documento: https://visiondefuturo.fce.unam.edu.ar/index.php/visiondefuturo/issue/view/18

ISSN 1668 - 8708 - Versión en Línea

E-mail: revistacientifica@fce.unam.edu.ar 
Guerrero, E. M., Guiñirgo, F. (2008). “Indicador espacial del metabolismo urbano. Huella Ecológica de la ciudad de Tandil, Argentina". Revibec: Revista Iberoamericana de Economía Ecológica, 9, 3144.

Iturbe, A., Guerrero, E. M. (2014). "Una aproximación a la Huella Ecológica de Malargüe, Argentina”. Revista Estudios Ambientales, 2(2), 39-57.

Lockhart, I.; Gómez, V. (2009). Cambio climático. Plan de acción Buenos Aires. 2030. Editorial Agencia de Protección Ambiental.

Mas, J. L., Rico, I. L. R., Nodal, P. M. (2012). “Cálculo de la Huella Ecológica en universidades cubanas. Caso de estudio: Universidad Central "Marta Abreu” de Las Villas”. Afinidad, 69(557), 30-34.

Tuia, D.; Ossés de Eicker, M.; Zah, R.; Osses, M.; Zarate, E., Clappier, A. (2007). "Evaluation of a simplified top-down model for the spatial assessment of hot traffic emissions in mid-sized cities". Atmospheric Environment, vol. 41, No. 17 (June), pp. 3658-3671.

Vicerrectorado de Campus y Sostenibilidad, Um. (2014). Huella ecológica de la Universidad de Málaga. Universidad de Málaga.

Wackernagel, M., Rees, W. E. (2001). Nuestra huella ecológica: reduciendo el impacto humano sobre la Tierra. LOM Ediciones, Santiago.

\section{RESUMEN BIOGRÁFICO}

\section{María José Fernandez}

Dra. en Economía (UBA). Investigadora Adjunta IIEP - Baires (UBA-CONICET). Prof. Adjunta FCE-UBA. Categoría Docente investigador III. Directora de Becas y Tesis. Integrante de la Comisión de Publicaciones. Secretaría de Investigación FCE-UBA. Codirectora CUADERNOS DEL CIMBAGE. Directora Proyecto UBACyT 2018. Intereses: Modelos alternativos para la Medición del Bienestar.

\section{Verónica García Fronti}

Doctora de la Universidad de Buenos Aires - Área Ciencias Económicas. Investigadora en el Centro de Investigación en Métodos Cuantitativos aplicados a la Economía y la Gestión (CMA - IADCOM). Profesora adjunta regular en la UBA. Intereses: Modelos matemáticos para gestión sobre la problemática del cambio climático.

\section{Andrea Parma}

Analista Universitaria de Sistemas, UTN. Profesora de Matemática y Astronomía, "INSP J.V. González". Prof. Adjunta Regular FCE-UBA. Vicerrectora Titular Escuela de Comercio № 16, "Gabriela Mistral", GCBA. Vocal Titular Junta de Disciplina MEIGC-GCBA. Investigadora Proyectos UBACYT, PROINC y PICT. Intereses: Matemática aplicada a las Ciencias Económicas.

\footnotetext{
"Visión de Futuro" Año 17, Volumen No 24 N² 2, Julio - Diciembre 2020 - Pág 125 - 145

URL de la Revista: http://visiondefuturo.fce.unam.edu.ar/index.php/visiondefuturo/index

URL del Documento: https://visiondefuturo.fce.unam.edu.ar/index.php/visiondefuturo/issue/view/18

ISSN 1668 - 8708 - Versión en Línea

E-mail: revistacientifica@fce.unam.edu.ar
} 